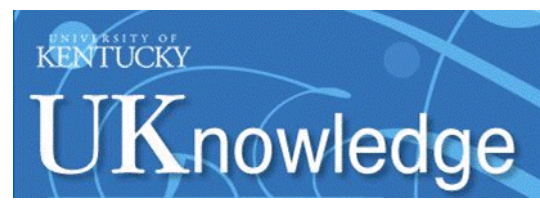

University of Kentucky

UKnowledge

$11-2009$

\title{
Aging Influences the Neural Correlates of Lexical Decision but not Automatic Semantic Priming
}

\author{
Brian T. Gold \\ University of Kentucky, brian.gold@uky.edu \\ Anders $\mathrm{H}$. Andersen \\ University of Kentucky, anders@mri.uky.edu \\ Greg A. Jicha \\ University of Kentucky, gregory.jicha@uky.edu \\ Charles D. Smith \\ University of Kentucky, csmith@mri.uky.edu
}

Follow this and additional works at: https://uknowledge.uky.edu/neurobio_facpub

Part of the Neuroscience and Neurobiology Commons

Right click to open a feedback form in a new tab to let us know how this document benefits you.

\section{Repository Citation}

Gold, Brian T.; Andersen, Anders H.; Jicha, Greg A.; and Smith, Charles D., "Aging Influences the Neural Correlates of Lexical Decision but not Automatic Semantic Priming" (2009). Neuroscience Faculty Publications. 6.

https://uknowledge.uky.edu/neurobio_facpub/6

This Article is brought to you for free and open access by the Neuroscience at UKnowledge. It has been accepted for inclusion in Neuroscience Faculty Publications by an authorized administrator of UKnowledge. For more information, please contact UKnowledge@lsv.uky.edu. 


\section{Aging Influences the Neural Correlates of Lexical Decision but not Automatic Semantic Priming}

\section{Digital Object Identifier (DOI)}

http://dx.doi.org/10.1093/cercor/bhp018

Notes/Citation Information

Published in Cerebral Cortex, v. 19, issue 11, p. 2671-2679.

The copyright holder has granted the permission for posting the article here. 


\section{Aging Influences the Neural Correlates of Lexical Decision but Not Automatic Semantic Priming}

Brian T. Gold ${ }^{1,2}$, Anders H. Andersen ${ }^{1,2}$, Greg A. Jicha ${ }^{3-5}$ and Charles D. Smith ${ }^{2-5}$

${ }^{1}$ Department of Anatomy and Neurobiology, ${ }^{2}$ Magnetic Resonance Imaging and Spectroscopy Center, ${ }^{3}$ Department of Neurology, ${ }^{4}$ Alzheimer's Disease Center and ${ }^{5}$ Sanders-Brown Center on Aging, Chandler Medical Center, University of Kentucky, Lexington, KY 40536, USA

\begin{abstract}
Human behavioral data indicate that older adults are slower to perform lexical decisions (LDs) than young adults but show similar reaction time gains when these decisions are primed semantically. The present study explored the functional neuroanatomic bases of these frequently observed behavioral findings. Young and older groups completed unprimed and primed LD tasks while functional magnetic resonance imaging (fMRI) was recorded, using a fully randomized trial design paralleling those used in behavioral research. Results from the unprimed task found that age-related slowing of LD was associated with decreased activation in perceptual extrastriate regions and increased activation in regions associated with higher level linguistic processes, including prefrontal cortex. In contrast to these age-related changes in brain activation, the older group showed a preserved pattern of fMRI decreases in inferior temporal cortex when LD was primed semantically. These findings provide evidence that older adults' LD abilities benefit from contexts that reduce the need for frontally mediated strategic processes and capitalize on the continued sensitivity of inferior temporal cortex to automatic semantic processes in aging.
\end{abstract}

Keywords: aging, fMRI, lexical decision, prefrontal cortex, priming, reaction time

\section{Introduction}

Visual word recognition involves the ability to determine whether a string of letters has a lexical status. This skill lies at the core of the human capacities for reading and comprehension of written language (Perfetti 1994). As such, a good deal of research has been conducted to explore how word recognition is affected by aging. Many such investigations have used the lexical decision (LD) task in which participants decide, as quickly as possible, if a string of letters is a word or a nonword. In general, this research has demonstrated that aging slows LD but does not affect its accuracy (Lima et al. 1991; Madden 1992; Myerson et al. 1992). Lexical tasks tend to undergo less agerelated slowing than tasks in some nonlexical cognitive domains (Lima et al. 1991). However, adult LD times have been found to slow consistently with increasing age, with reaction time (RT) increases of 4-10 ms/year (Madden 1992).

Models of visual word recognition characterize the LD task as an evidence-building process during which strategic processes operate until such a time when a lexical representation's threshold is reached and a response is executed (Ratcliff and Rouder 1998; Balota et al. 2004). However, strategic processes required to complete the LD task can be reduced substantially when the target word is preceded by a semantically related prime word (e.g., bread-butter) (Posner and Snyder 1975; Neely
1977). Under these conditions, older adults tend to show comparable semantic priming effect sizes as young adults (Howard 1983; Madden 1986; Ober et al. 1991). These findings suggest that older adults require more time to perform LDs than young adults under effortful conditions that emphasize strategic processes (unprimed conditions) but retain cognitive mechanisms that enable facilitation of LD (semantically primed conditions).

Despite the large body of behavioral data concerning agerelated changes in LD, knowledge concerning the neural bases of these age-related changes remains limited. However, a body of data do exist on the neural correlates of LD in young adults that can serve as a baseline from which to understand potential age-related changes. Imaging studies have demonstrated that young adults predominantly recruit left occipitotemporal and inferior frontal regions during visual LD (Price et al. 1994; Rumsey et al. 1997; Madden et al. 2002; Gold and Rastle 2007; Heim et al. 2009). There is evidence that different regions within this network play different roles in LD. Extrastriate regions have been linked with orthographic processes such as visual feature extraction and mapping visual patterns onto lexical representations (Petersen et al. 1990; Binder et al. 2003, 2006; Cohen et al. 2003; Gold et al. 2006). In contrast, inferior temporal regions have most typically been associated with semantic processes (Nobre et al. 1994; Martin et al. 1995; Martin and Chao 2001; Sharp et al. 2004), and left inferior frontal regions have been shown to contribute to multiple high-level linguistic processes (Petersen et al. 1988; Demb et al. 1995; Thompson-Schill et al. 1997; Badre et al. 2005; Gold et al. 2005, Gold et al. 2006).

Age-related slowing of LD could be associated with changes in the strength of activation of some occipitotemporal or inferior frontal regions typically activated in young adults. Alternatively, age-related slowing of LD could be associated with recruitment of regions not typically activated in young adults. In the present study, these different possibilities were explored through between-group comparisons of brain activation during the performance of an unprimed LD task. Following this comparison, we then attempted to determine whether any observed between-group functional magnetic resonance imaging (fMRI) activation differences contribute to age-related slowing of LD. This issue was explored through a series of regression analyses developed in the behavioral literature to assess how a mediating variable may contribute to the relationship between aging and RT (Salthouse 1993). First, multiple linear regression analyses were conducted to determine if the magnitude of activation in any region showing between-group activation differences was correlated with LD-RT. Second, hierarchical regression analyses were conducted to determine whether any region identified in the 
correlation analyses mediated the relationship between aging and LD-RT.

To investigate the effects of aging on the neural correlates of facilitated LD, participants also completed a task in which targets were preceded by primes that were either semantically related (e.g., bread-butter) or semantically unrelated (e.g., doctor-butter). A short duration of $250 \mathrm{~ms}$ between the prime and target (stimulus-onset asynchrony [SOA]) was employed in order to minimize strategy generation and attentional requirements and stress more automatic lexical-semantic facilitation processes. Semantic priming at short SOA is dominated by automatic processes because insufficient time is available to predict the target based upon the prime (Posner and Snyder 1975; Neely 1977). Instead, under short SOA conditions, LD is thought to be facilitated via a more passive spreading activation mechanism (Collins and Loftus 1975; Posner and Snyder 1975; Neely 1977). The neural correlates of automatic semantic priming were explored by looking for evidence of decreased fMRI activation on related trials compared with unrelated trials or fMRI priming, a finding thought to reflect increased efficiency in a particular processing domain (Mummery et al. 1999).

\section{Materials and Methods}

\section{Participants}

The research procedures were approved by the Institutional Review Board of the University of Kentucky Medical Center, and all participants provided informed written consent. Participants were 15 young adults ( 9 males, 6 females) between the ages of 19 and 26 years $(\mathrm{M}=22.9$, standard deviation $[\mathrm{SD}]=2.3)$ and 14 older adults ( 7 males, 7 females) between the ages of 64 and 83 years $(M=74.7$, $\mathrm{SD}=4.8$ ). The young and older groups did not differ in gender distribution $\left(\chi_{1}{ }^{2}=0.29, P=0.59\right)$ or mean number of years of education (younger group $\mathrm{M}=14.9, \mathrm{SD}=1.1$; older group $\mathrm{M}=15.4$, $\mathrm{SD}=1.6) \quad\left(t_{27}=1.1, P=0.27\right)$. All participants were communitydwelling individuals who were right-handed, with normal or corrected-to-normal visual acuity. Young adults were recruited from local advertisements. Older adults were recruited from the University of Kentucky Alzheimer's Disease Center (UK-ADC) longitudinal normal volunteer cohort. Inclusion criteria for this cohort are minimum 60 years of age, cognitive and neurological normality, agreement to brain donation to the UK-ADC at death, a designated informant for structured interviews, and willingness to undergo annual examinations. Participants are excluded from the cohort if they had a history of substance abuse (including alcohol), major head injury, major psychiatric illness, medical illnesses that are unstable and/or have an effect on the central nervous system, chronic infectious diseases, stroke or transient ischemic attack, encephalitis, meningitis, or epilepsy. An annual evaluation includes a comprehensive neuropsychological battery and general physical and neurological examinations that are detailed elsewhere (Schmitt et al. 2001). All older adults received a diagnosis of normal by the UK-ADC consensus conference within 3 months of their participation in the present study.

\section{Stimuli}

Word stimuli consisted of 360 semantically related prime-target noun pairs (e.g., spoon-fork), which were selected from a larger data set producing robust priming effects in previous research (Hutchison et al. 2008). Targets were high frequency $(M=109$ [Kucera and Francis $1967])$ and moderate length $(M=4.7$ letters $)$. Six lists of 60 semantically related word pairs were created in order to counterbalance stimuli across conditions. The mean frequency and length of the target words were matched across lists. Lists of unrelated pairs were generated from the related pair lists by pseudorandomly re-pairing targets and primes, with the constraint that the new pair did not share a semantic relationship. For the unprimed LD task, only targets from prime-target lists were used. Presentation of the matched lists were rotated across subjects such that primes and targets appeared an equal number of times as part of related and unrelated pairs and targets appeared an equal number of times in the unprimed and primed LD tasks. Pseudoword trials were also included to provide the "no" response for the LD tasks. Pseudoword targets were constructed by changing 1 or 2 letters of a group of word stimuli that were not used in the present experiment. Pseudoword targets were matched with word targets for length. Word primes used in the pseudoword target trials were matched for frequency and length with primes used in word target trials.

\section{Task Procedures}

Participants completed 2 kinds of visual LD tasks. In each LD task, participants were asked to decide if underlined letter strings were words or nonwords. Participants were asked to make their decisions as quickly and accurately as possible. In the unprimed LD task, trials consisted of a target for $1350 \mathrm{~ms}$ and a fixation cross (+) for $650 \mathrm{~ms}$. In the primed LD task, trials consisted of a prime for $250 \mathrm{~ms}$, a target for $1350 \mathrm{~ms}$, and a fixation cross (+) for $400 \mathrm{~ms}$. There were 3 runs for each of the LD task. The order of runs was counterbalanced across participants. The unprimed LD task had 2 trial types (word and pseudoword), and the primed LD task had 3 trial types (related, unrelated, and pseudoword). An event-related design was employed including trial types of interest and fixation trials (+) used for stimulus jittering. Different trial types were presented in a pseudorandom order and were separated from each other by a variable intertrial interval (range $=0-12 \mathrm{~s}$, $\mathrm{M}=2.8 \mathrm{~s}$ ) consisting of the fixation trials. Trial types were ordered for optimal experimental efficiency using optseq2 (http://surfer.nmr.mgh. harvard.edu/optseq/). Each run consisted of 120 trials. Unprimed LD runs had 60 word, 30 pseudoword, and 30 fixation trials. Primed LD runs had 30 related trials, 30 unrelated trials, 30 pseudoword trials, and 30 fixation trials.

Stimulus presentation and recording of responses were implemented with E-Prime software (Psychology Software Tools Inc, Pittsburgh, PA), using an MRI-compatible projection system (SilentVision SV-6011 LCD; Avotec Inc, Stuart, FL). Visual stimuli were projected onto a screen at the back of the magnet bore, viewed by subjects through a mirror mounted on the MR head coil. Stimuli were presented in black uppercase Courier font on a white background and subtended a vertical visual angle of about $1^{\circ}$ and an average horizontal visual angle of about $4.5^{\circ}$ at the center of the field of view (FOV). Responses were made via button presses, using a fiber-optic button box that registers latencies to the nearest millisecond. RTs were measured from the onset of target display. Behavioral RTs were computed for correct trials of each condition. Each participant's median RT for correct trials was entered into statistical analyses to minimize the effect of outliers.

\section{MRI Acquisition}

MRI data were collected on a 3-T Siemens Magnetom Trio MRI scanner. Foam padding was used to limit head motion within the coil. $\mathrm{T}_{2}{ }^{*}$ weighted functional images were acquired using the body coil to transmit and an 8-channel head array coil to receive. Main field $\mathrm{B}_{0}$ homogeneity was optimized at the start of each run using an automated shimming routine. Functional image runs were acquired in the transverse plane using a gradient-echo, echo-planar imaging (EPI) sequence (time echo $[\mathrm{TE}]=30 \mathrm{~ms}$, time repetition $[\mathrm{TR}]=2000 \mathrm{~ms}$, flip angle $\left.=77^{\circ}\right)$. Thirty-three interleaved slices were acquired $(64 \times 64$ image matrix, 224 $\times 224 \mathrm{FOV}$, with isotropic 3.5 - $\mathrm{mm}$ voxels), covering the entire cerebrum and the upper cerebellum. A double-echo gradient-echo sequence $\left(\mathrm{TE}_{1}=5.19 \mathrm{~ms}, \mathrm{TE}_{2}=7.65 \mathrm{~ms}\right)$ with slice position and spatial resolution matching those of the EPI acquisition was used to map the spatial inhomogeneity of the $\mathrm{B}_{0}$ field. A high-resolution, $3 \mathrm{D}$ anatomic image was acquired using a $T_{1}$-weighted (magnetization-prepared rapid gradient echo) sequence ( $\mathrm{TR}=2100 \mathrm{~ms}$, $\mathrm{TE}=2.93 \mathrm{~ms}$, time to inversion $[\mathrm{TI}]=1100 \mathrm{~ms}$, flip angle $=12^{\circ}, \mathrm{FOV}=224 \times 256 \times 192 \mathrm{~mm}, 1-\mathrm{mm}$ isotropic voxels, sagittal partitions) for the localization of functional activity in the stereotactic space of Talairach and Tournoux (1988). 


\section{fMRI Data Analysis}

fMRI data were analyzed with AFNI software (Cox 1996). Several preprocessing steps were employed to minimize artifacts. Following exclusion of the first few functional volumes (12 s), differences in timing between slices due to acquisition order were adjusted with sinc interpolation. Functional images were motion corrected and registered to the image collected closest in time to the high-resolution anatomical image using a 6-parameter rigid body transformation (Cox et al. 1999). Images were then unwarped via $\mathrm{B}_{0}$ field maps (using FSL software http://www.fmrib.ox.ac.uk/fsl) to reduce nonlinear magnetic field distortions. Finally, functional images were smoothed spatially with a 4 -mm root-mean-square Gaussian kernel and intensity normalized to yield subsequent activation measures expressed as percent signal change from baseline.

Deconvolution analysis was performed on each subject's preprocessed image time series to provide simultaneous parameter estimates of the hemodynamic response associated with each trial type (Glover 1999). The hemodynamic response was estimated at seven 2-s time lags (0-14 s) after stimulus presentation for correct trials in each condition compared with baseline fixation. Trials in which incorrect responses occurred were coded as a separate condition and included in the model as a nuisance covariate to increase statistical sensitivity. For the unprimed LD task, there were 14 regressors of interest ( 2 trial types $\times 7$ time lags). For the primed LD task, there were 21 regressors of interest ( 3 trial types $\times 7$ time lags). Each regressor consisted of a series of delta functions, producing an estimate of the response to each trial type, with no assumptions about the temporal dynamics of the hemodynamic response. Nuisance regressors included in the model were each run's mean, linear trend, and 6 outputs from volume registration to account for residual variance from subject motion. Each participant's regressor data sets were then transformed into standardized space using a combined young-older target. Participant's $T_{1}$-weighted images were first transformed into standard space (Talairach and Tournoux 1988) and then averaged using AFNI's 3dmerge program. Each participant's original $T_{1}$-weighted images in native space were then transformed to this merged young-old target. Participant's regressor data sets were then transformed to standard space, using landmarks from their anatomical data sets, and resampled at $2-\mathrm{mm}^{3}$ resolution using cubic spline interpolation.

During second-level analyses, group-based, voxelwise $t$-tests were performed on the mean of the 2 time points surrounding the peak activation in each condition (4-8 s from the 14-s estimated hemodynamic response). A mixed-effect model was employed that treated condition as a fixed effect and participants as a random effect. Each group's word and pseudoword trial types from the unprimed task were contrasted with visual fixation to identify the distributed network of regions involved in LD in young and older groups. In addition, unprimed LD activations of the 2 groups were compared directly.

Monte Carlo simulations were run using AlphaSim in order to determine the number of contiguous voxels (at $P<10^{-4}$ ) needed to be active in order to achieve a corrected significance level of $P<0.05$ for the unprimed LD comparisons. The Monte Carlo calculation used $10^{4}$ trials and indicated a minimum cluster size of 11 contiguous active voxels at the spatial resolution of the original EPI acquisition $(472 \mu \mathrm{L})$ This cluster threshold was applied to each group's unprimed LD activation map and the between-group comparison map. A conjunction approach was then used to identify fMRI priming effects (as reflected by decreased blood oxygen level-dependent response in the related compared with the unrelated condition). Each group's fMRI priming map (at $P<0.05$ ) was masked by its unprimed LD map to determine whether any regions within the distributed network involved in unprimed LD also showed fMRI priming associated with semantically facilitated components of word recognition. Activation maps were projected onto semi-inflated surfaces using Caret software (Van Essen et al. 2001).

\section{Region of Interest Analyses}

Time course and magnitude data were extracted from specific regions of interest (ROIs) activated in the whole-brain maps of primed and unprimed LD. ROI masks consisted of a 3D area including all contiguous voxels activated $(P<0.001)$ within $10 \mathrm{~mm}$ of a peak effect. Common ROI masks were used for each group for the unprimed LD task and were generated on the between-group wholebrain map (regions that showed age-group activation differences in unprimed LD). Group-specific masks were used for the primed LD task because no group differences were observed in fMRI priming. These ROI masks were generated to surround the peak effect in each group's whole-brain map of priming fixation. ROI masks were then applied to each subject's regressor data set to extract the average time course and magnitude of the hemodynamic response in each condition.

For the RT-fMRI analyses, a series of multiple regression analyses were conducted, with RT from the unprimed LD task serving as the outcome variable. In the multiple regression analyses, the independent variable was fMRI magnitude in ROIs corresponding to each of the regions that showed age-group activation differences (listed in Table 1). In the subsequent hierarchical regression analyses, the independent variables were age and fMRI magnitude in the mid left inferior prefrontal cortex (mid-LIPC) region showing a significant correlation with RT in the multiple regression analyses.

\section{Results}

\section{Bebavioral}

Both groups performed the unprimed LD task with very high accuracy ( $\mathrm{M}$ of young group $=96 \%, \mathrm{M}$ of older group $=98 \%$ ). The performance data of primary interest were correct RTs (Fig. 1). Overall, older adults had longer RTs than young adults $\left(F_{1,27}=37.84, P<0.0001\right)$, and RTs were longer on pseudoword than word trials $\left(F_{1,27}=187.96, P<0.0001\right)$. However, there was no group-by-trial-type interaction $\left(F_{1,27}=1.04\right.$, $P=0.32)$. Older adults had longer R'Ts than young adults on word trials $\left(t_{27}=5.02, P<0.0001\right)$ and pseudoword trials $\left(t_{27}=6.13\right.$, $P<0.0001)$.

Results from the primed LD task indicated age-related slowing on both unrelated and related trial types. Overall, older adults had longer RTs than young adults $\left(F_{1,27}=34.43\right.$, $P<0.0001$ ), and the 3 kinds of trials (related, unrelated, pseudoword) resulted in different RTs $\left(F_{2,54}=175.22, P<\right.$ $0.0001)$. However, there was no group-by-trial-type interaction $\left(F_{2,54}=1.02, P=0.37\right)$. Older adults had longer RTs than young adults on related $\left(t_{27}=4.99, P<0.0001\right)$, unrelated $\left(t_{27}=4.93\right.$, $P<0.0001)$, and pseudoword trials $\left(t_{27}=7.26, P<0.0001\right)$.

Despite the observed age-related slowing on LD tasks, the magnitude of semantic priming effects (Fig. 1, panel $C$ ) was not different between groups $\left(t_{27}=0.40, P=0.69\right)$. Both groups showed highly significant RT priming effects (young: $t_{13}=5.65$, $P<0.0001$; older: $\left.t_{14}=7.61, P<0.0001\right)$.

\begin{tabular}{|c|c|c|c|c|c|}
\hline \multicolumn{6}{|l|}{$\begin{array}{l}\text { Table } 1 \\
\text { Talairach and Tournoux active }\end{array}$} \\
\hline Region & $\sim \mathrm{BA}$ & $x$ & y & $z(\mathrm{~mm})$ & Cluster ( $\mu \mathrm{L})$ \\
\hline \multicolumn{6}{|l|}{ Young $>$ old } \\
\hline Left middle occipital gyrus & 18 & -29 & -83 & 0 & 3080 \\
\hline Left lingual gyrus & 18 & -26 & -80 & -6 & 2771 \\
\hline Right middle occipital gyrus & 18 & 33 & -80 & -7 & 2014 \\
\hline Right lingual gyrus & $18 / 19$ & 8 & -81 & -8 & 984 \\
\hline \multicolumn{6}{|l|}{ Old $>$ young } \\
\hline Left fusiform gyrus & 37 & -38 & -59 & -13 & 3207 \\
\hline Left inferior temporal gyrus & $21 / 37$ & -46 & -67 & 0 & 3023 \\
\hline Left inferior frontal gyrus & $45 / 44$ & -45 & 21 & 10 & 476 \\
\hline
\end{tabular}

Note: $\mu \mathrm{L}=$ volume. Clusters from Figure 3 of $\geq 472 \mu \mathrm{L}$ are listed. 


\section{Functional Magnetic Resonance Imaging}

Figure 2 presents activations associated with unprimed LD in each group. In the young group (top panel), LD was associated with activation of a network of regions, prominently involving ventral occipitotemporal cortex and anterior cingulate bilaterally, and LIPC. The older group (bottom panel) activated a similar network of occipitotemporal, cingulate, and LIPC regions.

Results from the between-group comparison of unprimed LD are presented in Figure 3. All between-group activation differences meeting the uncorrected significance level of $P<$ 0.001 are presented in order to appreciate the continuity of the data. However, only clusters meeting a corrected significance level of $P<0.05$ (those with a volume $\geqslant 472 \mu \mathrm{L}$ ) are interpreted (Table 1). The younger group showed increased activation compared with the older group in several regions of extrastriate cortex, with the largest increases in the left hemisphere. In contrast, the older group showed increased activation compared with the younger group in the left fusiform gyrus (FFG), the left inferior temporal gyrus, and a small portion of mid-LIPC.

\section{RT-fMRI Correlations}

Multiple regression analyses were conducted to determine whether any of the regions showing age-related activation differences (in the above analyses) showed a relationship between unprimed LD-RT and fMRI response magnitude. The
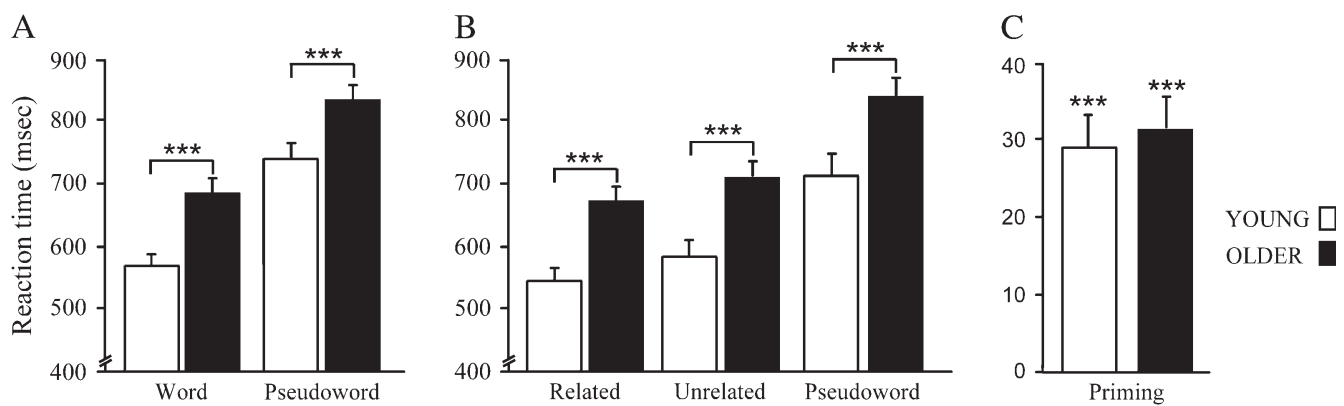

Figure 1. RT results from the unprimed and primed LD tasks. (A) Mean group RTs to word and pseudoword trials of the unprimed LD task. $(B)$ Mean group RTs to the related, unrelated, and pseudoword trials of the primed LD task. $(C)$ Mean group priming effects (unrelated-related). Note: ${ }^{* *} P<0.0001$.
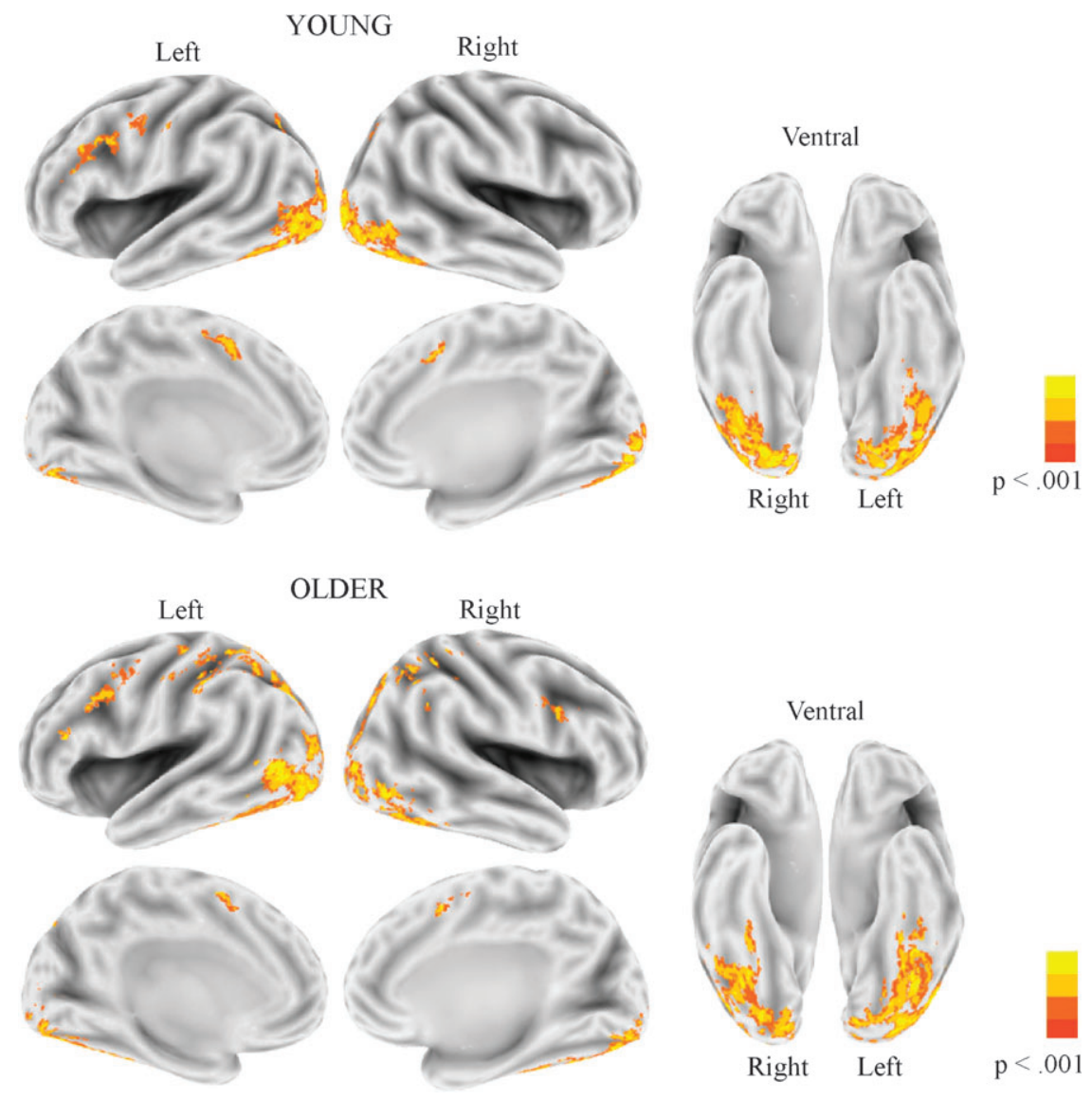

Figure 2. Brain activations associated with unprimed LD. Whole-brain LD activation maps (LD-fixation) in the young (top) and older (bottom) groups. The level of significance is indicated by the color bar at the right side. A significance threshold of $P<0.001$ was used. 

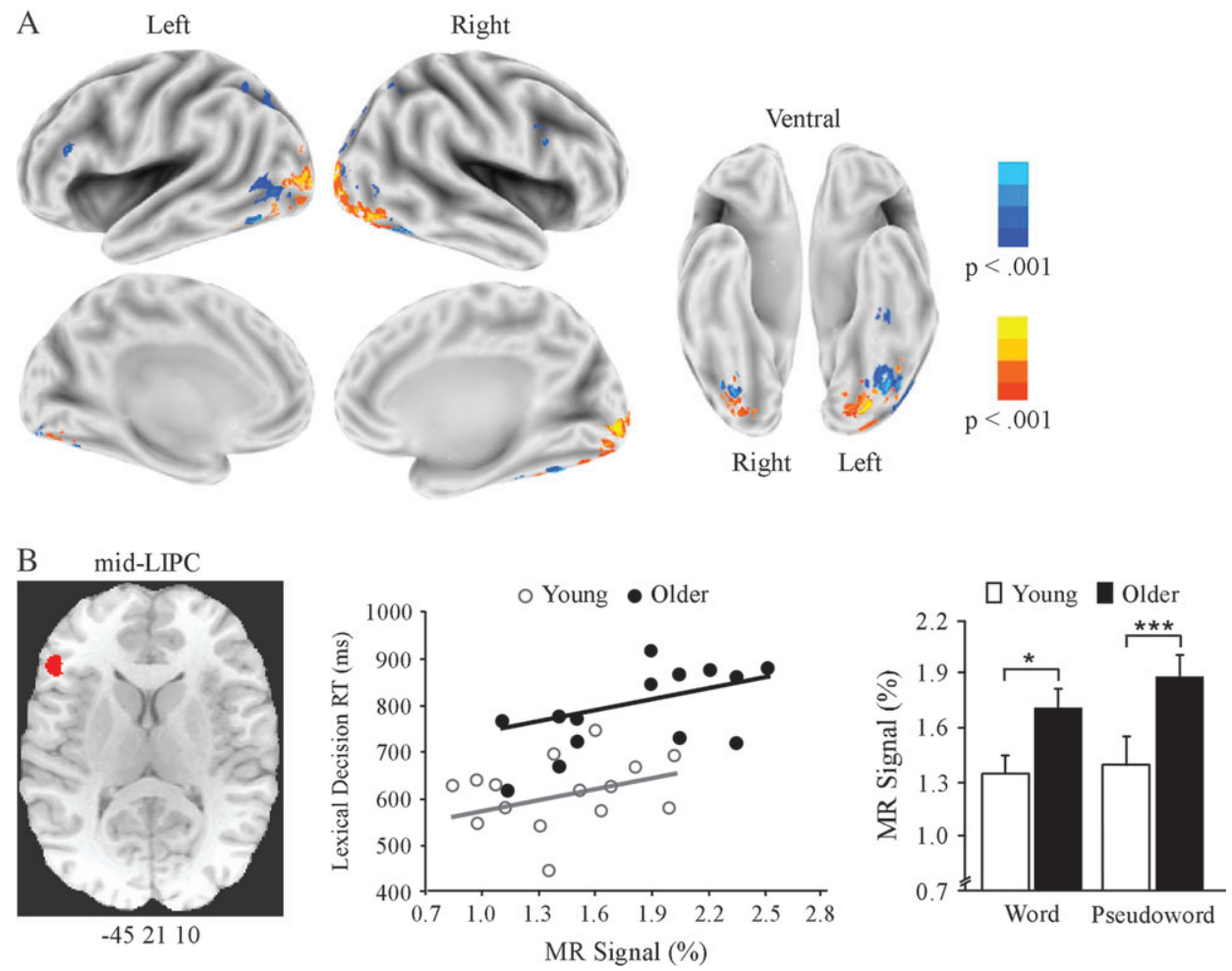

Figure 3. Between-group results from the unprimed LD task. (A) Whole-brain group activation differences. Warm colors (red-yellow) indicate regions showing increased activation in the young group, and cold colors (blue) indicate regions showing increased activation in the older group. The level of significance is indicated by the color bar at the right side. All between-group activation differences meeting the uncorrected significance level of $P<0.001$ are presented in order to appreciate the continuity of the data. However, only clusters meeting a corrected significance level of $P<0.05$ (those with a volume $\geq 472 \mu \mathrm{L}$ ) are interpreted (Table 1). (B) The left panel provides a representation of the mid-LIPC ROI overlaid on a high-resolution image of a single subject in standardized space. Peak Talairach coordinates are given under the image. The middle panel shows regression plots of the significant correlations between unprimed LD-RT and fMRI magnitude in the mid-LIPC region in age-group. Each circle represents one participant. The right panel shows bar charts of the mean MR percentage signal change from fixation in the mid-LIPC region for the word and pseudoword trial types in each age-group.

multiple regression model for the young group indicated a relationship between unprimed LD-RT and fMRI magnitude in the mid-LIPC (peak coordinates: $-45,21,10)(F=2.4, P<0.05)$. There was a slight trend for a relationship between LD-RT and fMRI magnitude in the left lingual gyrus (peak coordinates: $-26,-80,-60)(F=1.6, P<0.13)$. The remaining regressors were uncorrelated with RT ( $\operatorname{sex}[P=0.64]$, all other ROIs $[P \geqslant$ 0.37]). The model for older adults also revealed a correlation between RT and fMRI magnitude in the left IFG $(F=2.7, P<$ $0.05)$. The remaining regressors were uncorrelated with RT (sex $[P=0.63]$, all other ROIs $[P \geqslant 0.28]$ ). Figure 3 (panel $B$ ) presents the regression plot of the significant RT-fMRI-positive correlation in the mid-LIPC for both the young group $\left(r_{15}=0.51, P<0.05\right)$ and the older group $\left(r_{14}=0.54, P<\right.$ $0.05)$. Younger and older individuals with relatively slow RT tended to show increased recruitment of mid-LIPC.

\section{Comparison of Word and Pseudoword Activations in Mid-LIPC}

A 2-way analysis of variance (ANOVA) was conducted with midLIPC response as the dependent variable to determine whether the age-related increase in mid-LIPC interacted with stimulus type (words vs. pseudowords). The results are presented in Figure 3 (panel $C$ ). Overall, the older group showed a higher mid-LIPC response than the young group $\left(F_{1,27}=9.3, P<\right.$ $0.001)$. There was a slight trend for a higher mid-LIPC response during pseudoword trials than word trials $\left(F_{1,27}=2.7, P<0.11\right)$. There was an age-group $\times$ stimulus type interaction in mid-
LIPC response because the older group showed a larger increase than the young group during pseudoword trials compared with word trials $\left(F_{1,27}=4.3, P<0.05\right)$. However, the age-related increase in mid-LIPC response was evident for both word trials $\left(t_{27}=2.6, P<0.05\right)$ and pseudoword trials $\left(t_{27}=3.6\right.$, $P<0.001)$.

\section{Mediation Analysis}

The above analyses determined that the mid-LIPC region showing age-related increases in unprimed LD activation also shows a pattern of response magnitude that is correlated with RT. Together, these findings raised the possibility that agerelated mid-LIPC activation increases during LD may contribute to age-related increases in RT. Hierarchical regression analyses predicting LD-RT were conducted to explore this possibility (Table 2). In model 1 , when age-group was the only predictor, $38 \%$ of the variance in LD-RT was age related. In model 2, when mid-LIPC magnitude was entered first, followed by age-group, mid-LIPC magnitude was a significant predictor, accounting for $26 \%$ of the variance in LD-RT. After controlling for mid-LIPC magnitude, age-group still accounted for significant variance in LD-RT. However, the relationship between age and LD-RT was significantly reduced when fMRI magnitude in the mid-LIPC was taken into account.

In order to assess the degree to which fMRI magnitude in the mid-LIPC attenuated the amount of variance in LD-RT that can be explained by age, we followed a procedure suggested by Salthouse (1993), in which we subtracted the amount of 
variance in LD-RT uniquely associated with age (when magnitude in the mid-LIPC was included in the model) from the amount of variance in LD-RT associated with age as a sole predictor. We then divided this difference by the amount of age-related variance in LD-RT when age was the sole predictor. Results indicated that fMRI magnitude in the mid-LIPC attenuated $53 \%$ of the age-related variance in LD-RT. These results demonstrate that the older group's overrecruitment of mid-LIPC contributes to its increased LD-RT.

Figure 4 presents fMRI priming results. Whole-brain analyses indicated that both young and older groups showed robust fMRI priming in inferior temporal cortex. A direct comparison of fMRI priming was also conducted and revealed no group differences. In each group, fMRI priming was biliateral but more extensive in the left hemisphere. Also in each group, the largest fMRI priming effect was observed in left mid-FFG

\begin{tabular}{|c|c|c|c|c|c|}
\hline \multicolumn{6}{|c|}{$\begin{array}{l}\text { Table } 2 \\
\text { Effects of age and mid-LIPC magnitude on LD-RT }\end{array}$} \\
\hline & $r^{2}$ & $\begin{array}{l}\text { Increment } \\
\text { in } r^{2}\end{array}$ & $F$ & Attenuation & $\%$ Attenuation \\
\hline \multicolumn{6}{|l|}{ Model 1} \\
\hline $\begin{array}{l}\text { Age (years) } \\
\text { Model } 2\end{array}$ & 0.38 & & $16.50^{* * *}$ & & \\
\hline fMRI-LIPC & 0.26 & & $9.69^{* *}$ & & \\
\hline Age (years) & 0.44 & 0.18 & $8.15^{* *}$ & 0.20 & 52.63 \\
\hline
\end{tabular}

${ }^{* *} P<0.01,{ }^{* * *} P<0.001$.
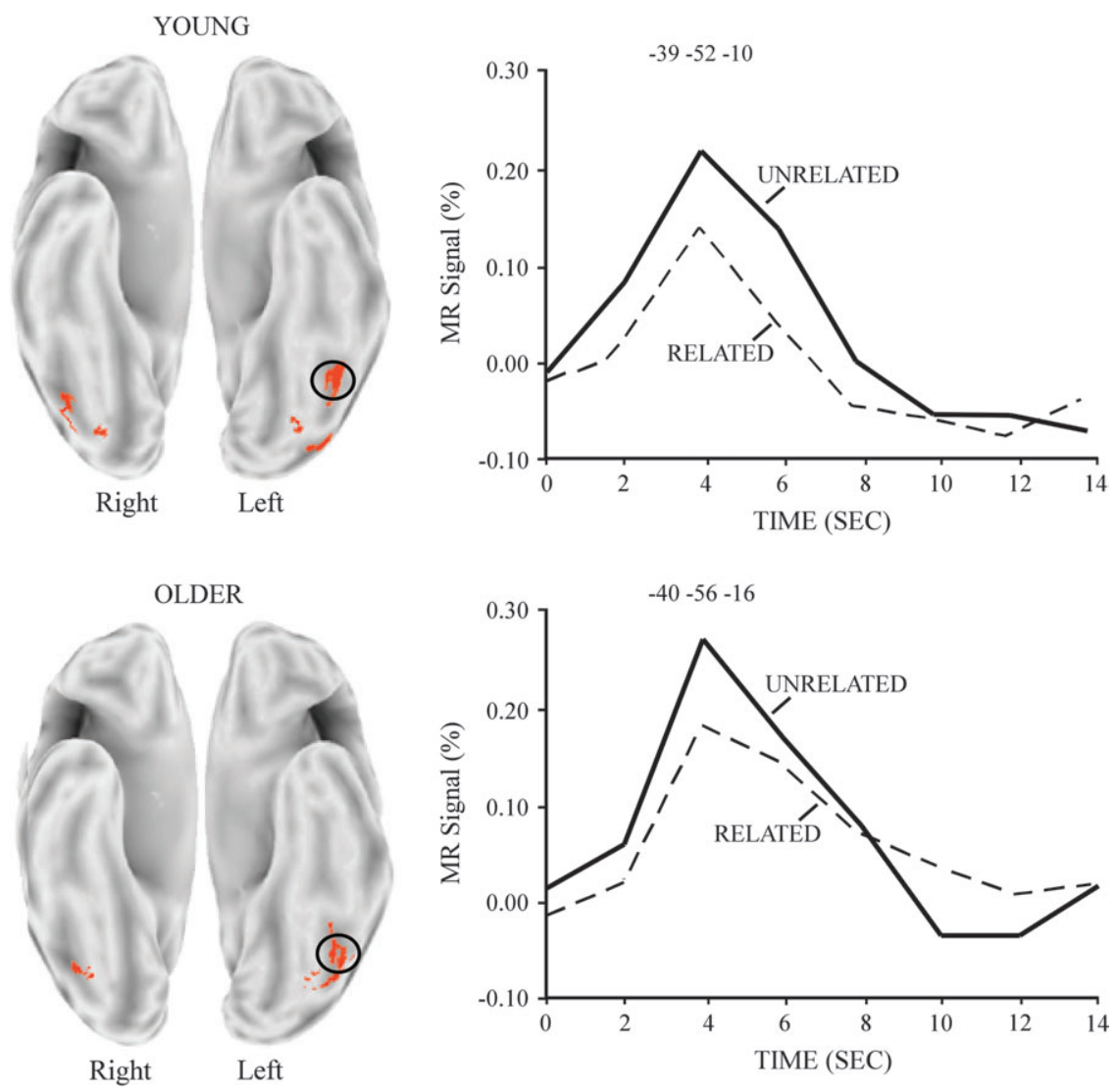

Figure 4. fMRI Priming effects. The left panel presents maps of decreased activations in the related condition compared with the unrelated condition for each group. Each group's fMRI priming map was thresholded at $P<0.05$ and masked by activation in their unprimed LD map (see Fig. 2). The circled region is the mid-FFG region that showed fMRI priming in each group. The right panel displays the time course of activation for unrelated and related trial types in mid-FFG. The peak Talairach coordinates of the mid-FFG priming effect are given above the time courses. (young peak: $-39,-52,-10$; older peak: $-40,-56,-16$ ). A 2 -factor mixed ANOVA was run with mean activation magnitudes in mid-FFG as the dependent variable, trial type (unrelated and related) as the within-subjects factor, and age-group (young and older) as a between-subjects factor. There was a significant effect of trial type $\left(F_{1,27}=14.80, P<0.001\right)$ and a significant effect of group $\left(F_{1,27}=4.35, P<0.05\right)$ on mid-FFG magnitudes. However, there was no trial-type-by-group interaction $\left(F_{1,27}=\right.$ $0.19, P=0.67)$. Post hoc tests revealed that the older group had higher mid-FFG magnitudes on the unrelated trials $\left(t_{27}=\right.$ 2.08, $P<0.05)$ and marginally higher mid-FFG magnitudes on the related trials $\left(t_{27}=1.99, P=0.056\right)$. These results indicate that, although older adults maintain a higher response than young adults in mid-FFG on both unrelated and related LD trials (consistent with results from the unprimed LD task), they show comparable fMRI priming (unrelated-related) in this region.

\section{Discussion}

Behavioral results observed age-related slowing of LD, consistent with previous findings (Lima et al. 1991; Madden 1992; Myerson et al. 1992). Also consistent with previous findings, older adults showed comparable behavioral semantic priming effect sizes as young adults (Howard 1983; Madden 1986; Ober et al. 1991). The present study explored the functional neuroanatomic correlates of these frequently observed behavioral findings. Results from the unprimed task found that the older group's slowed LD was associated with underrecruitment 
of perceptual extrastriate regions and overrecruitment of regions associated with higher level linguistic processes. In contrast, results from the primed LD task link preserved automatic semantic priming in older adults with a preserved pattern of fMRI priming in inferior temporal cortex. Below we discuss these findings and their implications.

In both groups, unprimed LD was associated with activation of a network of occipitotemporal regions and LIPC. Age-related changes were observed in the strength of activation of several regions within this network. Specifically, older adults showed decreased activation compared with young adults in portions of extrastriate cortex (ca. Brodmann's area [BA] 18/19) and increased activation in inferior temporal cortex (ca. BAs 21, 37/ 21) and mid-LIPC (ca. BA 45/44). The finding of an age-related shift in the degree of activation from posterior to anterior regions during $\mathrm{LD}$ is generally in keeping with results from 2 previous positron emission tomography imaging studies that have compared young and older adults (Madden et al. 1996, 2002). Madden et al. (1996) reported decreased LD activation in older adults in extrastriate cortex (ca. BA 18). Madden et al. (2002) observed age-related decreases in extrastriate cortex (ca. BA 17) accompanied by age-related increases in inferior temporal cortex (ca. BA 37).

The tendency of older adults to underrecruit extrastriate regions and overrecruit inferior temporal cortex and mid-LIPC suggests that they may rely less on orthographic processes during LD than younger adults and more on higher level linguistic processes. This possibility is consistent with previous behavioral results suggesting that orthographic processes are more affected by aging than lexical-semantic processes. For example, degrading stimulus quality increases RT more in older adults than younger adults, whereas the effects of varying word frequency remain relatively consistent in aging (Madden 1988; Allen et al. 1995). In addition, at the functional neuroanatomic level, extrastriate regions showing greater activation in the young group have been associated with orthographic processes (Petersen et al. 1990; Cohen et al. 2003; Binder et al. 2003, 2006). In contrast, inferior temporal regions showing greater activation in the older group have typically been associated with semantic knowledge (Nobre et al. 1994; Martin et al. 1995; Martin and Chao 2001; Sharp et al. 2004), and the midLIPC region (ca. BA 45/44) showing greater activation in the older group had been found to contribute to high-level linguistic control processes across multiple verbal task domains (Thompson-Schill et al. 1997; Badre et al. 2005; Gold et al. 2005, 2006).

Although most data concerning the function of mid-LIPC have come from research conducted with young participants, results from the RT-fMRI analyses suggest that this region continues to contribute to strategic control processes in aging. In both groups, LD-RT was positively correlated with magnitude of activation in mid-LIPC. In other words, both young and older individuals with relatively long LD-RTs tended to recruit mid-LIPC more than those age-matched peers with shorter LD-RTs. The finding that both groups showed a correlation between LD-RT and magnitude of activation in mid-LIPC (but not other regions such as the inferior temporal region showing sensitivity to automatic semantic priming) is in keeping with a view that mid-LIPC contributes to strategic control processes in older adults as well as young adults because control processes have been shown to evolve more slowly than automatic processes (Neely 1977; Schneider and Shiffrin 1977). The activation pattern of a region contributing to control processes would thus be more likely than one associated with automatic processes to be positively correlated with RT, and this regionbased dissociation was evident in both age-groups.

Results from the hierarchical regression analyses demonstrated that age-related overrecruitment of this mid-LIPC region was associated with age-related increases in LD-RT. The agerelated overrecruitment of mid-LIPC, and increased LD-RT, may represent an attempt at compensation. For example, the influential model of Balota and Chumbley (1984) suggests that making an LD consists of orthographic familiarity processes and more attention demanding, strategic processes. The orthographic processes can be used to reach a quick LD in cases where the stimulus seems either very familiar or unfamiliar. When this process is insufficient to reach a word/nonword decision, a second set of strategic, and more time consuming, processes are initiated. The age-related overrecruitment of midLIPC may in part reflect increased emphasis by older adults on strategic processes in an attempt to compensate for their underrecruitment of extrastriate regions (which have been linked with faster orthographic processes).

The age-related overrecruitment of mid-LIPC during LD was observed for word and pseudoword trials and was most pronounced for pseudoword trials. This suggests that agerelated overrecruitment of mid-LIPC appears to relate to strategic processes involved in making word/nonword decisions, as opposed to word recognition per se. Strategic processes involved in LD, which may be particularly emphasized during correct rejection of pseudowords, may include checking the spelling of the stimulus against a spelling held in long-term memory (Balota and Chumbley 1984), sounding out the stimulus to assess phonological familiarity (Perfetti 1999), or consulting the semantic system (Plaut et al. 1996). One possible interpretation is that the older group's overrecruitment of midLIPC reflects an accuracy-speed trade-off during LD. For example, increased recruitment of a region linked with strategic processes could not only aid older adults in reaching a word/nonword decision but also would be likely to prolong their RT due to the slower nature of these processes compared with orthographic familiarity processes.

However, the possibility that age-related overrecruitment of mid-LIPC reflects a speed accuracy-speed trade-off in LD must be considered speculative because the older group's performance was at ceiling, precluding an analysis of the relationship between fMRI activation and accuracy. It is therefore also possible that the age-related increase in mid-LIPC response represents a failed attempt at compensation. For example, agerelated overrecruitment of mid-LIPC may reflect an attempt to compensate for decreased anatomical connectivity between posterior and anterior regions in the distributed word recognition network. There is evidence that white matter microstructure in both posterior and anterior regions contribute to LD-RT (Gold et al. 2007). This variable was not explored in the present study but deserves future analysis as a potential contributing factor to the present results. In addition, future studies could explore whether an age-related increase in mid-LIPC response actually helps older adults reach a word/ nonword decision by including more difficult stimuli, such as very low-frequency words and pseudohomophone nonwords. Making the LD task more difficult would increase the variance in accuracy, enabling correlational analysis between accuracy and fMRI activation. 
In contrast to the robust age-related activation changes associated with unprimed LD, older adults showed similar neural correlates of semantically facilitated LD as young adults. In each group, fMRI priming was observed in portions of the larger inferior temporal region activated during the unprimed LD task. Also in each group, the largest focus of fMRI priming was observed in the mid-FFG of the left hemisphere. The peak coordinates of fMRI priming effect were close to those previously reported in 2 previous imaging studies involving young adults (Wheatley et al. 2005; Gold et al. 2006), demonstrating the reproducibility of the fMRI priming associated with the automatic semantic priming behavioral effects. These results demonstrate that older adults can show a preserved fMRI response pattern during LD when the task is automated via the presentation of a brief semantic prime.

At first pass, the finding that mid-FFG was the region showing fMRI priming in the older group appeared somewhat surprising, given that this region was part of a larger inferior temporal area that showed age-related increases during the unprimed LD task. Insight into this apparent discrepancy was gained by examining mid-FFG activations separately for unrelated and related trial types. These analyses observed that the older group showed increased mid-FFG activation compared with the young group in both the unrelated and related trial types. However, analyses comparing these trial types indicated that the relative difference between them (fMRI priming) in mid-FFG was minimally affected by age (in terms of spatial extent and magnitude). Taken together, data from the unprimed and primed tasks indicate that the influence of age on mid-FFG response is context dependent: older adults appear to maintain a higher response in mid-FFG during unprimed LD (possibly reflecting increased semantic contribution) but show preserved fMRI decreases in this region when LD is facilitated through automatic semantic priming.

It is noteworthy that fMRI priming effects were observed in inferior temporal cortex but not in LIPC. A previous study reported that older adults showed preserved neural correlates of repetition priming in LIPC (Lustig and Buckner 2004). The different anatomical foci of these findings likely relate to the differing emphasis on automatic versus strategic processes. Repetition priming can be based in part upon learning of stimulusresponse associations and can thus be susceptible to strategic inhibitory effects (Dobbins et al. 2004). In contrast, semantic priming at short SOA such as the one used here ( $\mathrm{SOA}=250 \mathrm{~ms}$ ) is thought to be dominated by automatic facilitation processes because insufficient time is available to predict the target based upon the prime (Posner and Snyder 1975; Neely 1977). The most widely accepted behavioral explanation of the mechanism underlying semantic priming at short SOA is passive spreading activation mechanism whereby activation from the prime word spreads to related nodes within semantic memory, resulting in shorter RT to related targets due to a reduced recognition threshold (Collins and Loftus 1975; Posner and Snyder 1975; Neely 1977). The present results suggest that this automatic form of priming remains intact in aging in part due to the continued sensitivity of mid-FFG to implicit semantics.

In summary, results from the present study provide evidence for functional neuroanatomic correlates of 2 established behavioral findings in the word recognition literature: age-related slowing of unprimed LD and age-related preservation of semantically primed LD. Results demonstrate that age-related slowing of LD is associated with underrecruitment of extras- triate cortex and overrecruitment of inferior temporal inferior temporal cortex and mid-LIPC. In contrast to these activation differences, results link preserved automatic semantic components of word recognition in aging with a preserved pattern of fMRI priming in mid-FFG. Taken together, these results suggest that older adults' ability for speeded LD benefits from contexts that reduce the need for frontally mediated strategic processes and capitalize on the continued sensitivity of inferior temporal cortex to automatic semantic components of word recognition in aging.

\section{Funding}

National Institutes of Health (DC007315, P50 AG05144-21).

\section{Notes}

The authors thank Rickey Pack for help with data collection and Dr David Powell for technical support. We also thank Dr Michael Cortese for helpful comments. Conflict of Interest: None declared.

Address correspondence to Brian T. Gold, Department of Anatomy and Neurobiology, Chandler Medical Center, University of Kentucky School of Medicine, Lexington, KY 40536-0298, USA. Email: brian. gold@uky.edu.

\section{References}

Allen PA, Madden DJ, Slane SD. 1995. Visual word recognition and the effect of adult age and word frequency. In: Allen PA, Bashore TR, editors. Age differences in word and language processing. Amsterdam (The Netherlands): North-Holland. p. 30-71.

Badre D, Poldrack RA, Paré-Blagoev EJ, Insler RZ, Wagner AD. 2005. Dissociable controlled retrieval and generalized selection mechanisms in ventrolateral prefrontal cortex. Neuron. 47:907-918.

Balota DA, Chumbley JI. 1984. Are lexical decisions a good measure of lexical access? The role of word frequency in the neglected decision stage. J Exp Psychol Hum Percept Perform. 10:340-357.

Balota DA, Cortese MJ, Sergent-Marshall SD, Spieler DH, Yap M. 2004. Visual word recognition of single-syllable words. J Exp Psychol Gen. 133:283-316.

Binder JR, McKiernan K, Parsons ME, Westbury CF, Possing ET, Kaufman JN, Buchanan L. 2003. Neural correlates of lexical access during visual word recognition. J Cogn Neurosci. 15:372-393.

Binder JR, Medler DA, Westbury CF, Liebenthal E, Buchanan L. 2006. Tuning of the human left fusiform gyrus to sublexical orthographic structure. NeuroImage. 33:739-748.

Cohen L, Martinaud O, Lemer C, Lehericy S, Samson Y, Obadia M, Slachevsky A, Dehaene S. 2003. Visual word recognition in the left and right hemispheres: anatomical and functional correlates of peripheral alexias. Cereb Cortex. 12:1313-1333.

Collins AM, Loftus EF. 1975. A spreading-activation theory of semantic processing. Psychol Rev. 82:407-428.

Cox RW. 1996. AFNI: software for analysis and visualization of functional magnetic resonance neuroimages. Comput Biomed Res. 29: 162-173.

Cox RW, Jesmanowicz A. 1999. Real-time 3D image registration for functional MRI. Magn Reson Med. 42:1014-1018.

Demb JB, Desmond JE, Wagner AD, Vaidya CJ, Glover GH, Gabrielli JDE. 1995. Semantic encoding and retrieval in the left inferior prefrontal cortex: a functional MRI study of task difficulty and process specificity. J Neurosci. 15:5870-5878.

Dobbins IG, Schnyer DM, Verfaellie M, Schacter DL. 2004. Cortical activity reductions during repetition priming can result from rapid response learning. Nature. 428:316-319.

Glover GH. 1999. Deconvolution of impulse response in event-related BOLD fMRI. NeuroImage. 9:416-429.

Gold BT, Balota DA, Jones SJ, Powell DK, Smith CD, Andersen AH. 2006. Dissociation of automatic and strategic lexical-semantics: functional magnetic resonance imaging evidence for differing roles of multiple frontotemporal regions. J Neurosci. 26:6523-6532. 
Gold BT, Balota DA, Kirchhoff BA, Buckner RL. 2005. Common and dissociable activation patterns associated with controlled semantic and phonological processing: evidence from fMRI adaptation. Cereb Cortex. 15:1438-1450.

Gold BT, Powell DK, Xuan L, Jiang Y, Hardy PA. 2007. Speed of lexical decision correlates with diffusion anisotropy in left parietal and frontal white matter: Evidence from diffusion tensor imaging. Neuropsychologia. 45:2439-2446.

Gold BT, Rastle K. 2007. Neural correlates of morphological decomposition during visual word recognition. J Cogn Neurosci. 19:1983-1993.

Heim S, Eichhoff SB, Ischebeck AK, Friederici AD, Stephan KE, Amunts K. 2009. Effective connectivity of the left BA 44, BA 45 and inferior temporal gyrus during lexical and phonological decisions identified with DCM. Hum Brain Mapp. 30:392-402

Howard DV. 1983. The effects of aging and degree of association on the semantic priming of lexical decisions. Exp Aging Res. 9: 145-151.

Hutchison KA, Balota DA, Cortese MJ, Watson MJ. 2008. Predicting semantic priming at the item level. Q J Exp Psychol. 61:1036-1066.

Kucera H, Francis WN. 1967. Computational analysis of present-day American English. Providence (RI): Brown University Press.

Lima SD, Hale S, Myerson J. 1991. How general is general slowing? Evidence from the lexical domain. Psychol Aging. 6:416-425.

Lustig C, Buckner RL. 2004. Preserved neural correlates of priming in old age and dementia. Neuron. 42:865-875.

Madden DJ. 1986. Adult age differences in visual word recognition: semantic encoding and episodic retention. Exp Aging Res. 12:71-78.

Madden DJ. 1988. Adult age differences in the effects of sentence context and stimulus degradation during visual word recognition. Psychol Aging. 3:167-172.

Madden DJ. 1992. Four to ten milliseconds per year: age-related slowing of visual word identification. J Gerontol. 47:59-68.

Madden DJ, Langley LK, Denny LL, Turkington TG, Provenzale JM, Hawk TC, Coleman RE. 2002. Adult age differences in visual word identification: functional neuroanatomy by positron emission tomography. Brain Cogn. 49:297-321.

Madden DJ, Turkington TG, Coleman RE, Provenzale JM, DeGrado TR, Hoffman JM. 1996. Adult age differences in regional cerebral blood flow during visual world identification: evidence from H215O PET. NeuroImage. 3:127-142.

Martin A, Chao LL. 2001. Semantic memory and the brain: structure and processes. Curr Opin Neurobiol. 11:194-201.

Martin A, Haxby JV, Lalonde FM, Wiggs CL, Ungerleider LG. 1995. Discrete cortical regions associated with knowledge of color and action, regions associated with knowledge of color and action. Science. 270:102-105.

Mummery CJ, Shallice T, Price CJ. 1999. Dual-process model in semantic priming: a functional neuroimaging perspective. NeuroImage. 9:516-525.

Myerson J, Ferraro FR, Hale S, Lima SD. 1992. General slowing in semantic priming and word recognition. Psychol Aging. 7:257-270.

Neely JH. 1977. Semantic priming and retrieval from lexical memory: roles of inhibitionless spreading activation and limited-capacity attention. J Exp Psychol Gen. 106:226-254.
Nobre AC, Allison T, McCarthy G. 1994. Word recognition in the human inferior temporal lobe. Nature. 372:260-263.

Ober BA, Shenaut GK, Jagust WJ, Stillman RC. 1991. Automatic semantic priming with various category relations in Alzheimer's disease and normal aging. Psychol Aging. 6:647-660.

Perfetti CA. 1994. Psycholinguistics and reading ability. In: Gernsbacher MA, editor. Handbook of psycholinguistics. San Diego (CA): Academic Press. p. 849-894.

Perfetti CA. 1999. Comprehending written language: a blueprint of the reader. In: Brown CM, Hagoort P, editors. The neurocognition of language. Oxford: Oxford University Press. p. 167-208.

Petersen SE, Fox PT, Posner MI, Mintun M, Raichle ME. 1988. Positron emission tomographic studies of the cortical anatomy of singleword processing. Nature. 331:585-589.

Petersen SE, Fox PT, Snyder AZ, Raichle ME. 1990. Activation of extrastriate and frontal cortical areas by visual words and word-like stimuli. Science. 249:1041-1044.

Plaut DC, McClelland JL, Seidenberg MS, Patterson K. 1996. Understanding normal and impaired word reading: computational principles in quasi-regular domains. Psychol Rev. 103:56-115.

Posner MI, Snyder CRR. 1975. Facilitation and inhibition in the processing of signals. In: Rabbitt PMA, editor. Attention and performance V. London: Academic Press.

Price CJ, Wise RJ, Watson JD, Patterson K, Howard D, Frackowiak RS. 1994. Brain activity during reading. The effects of exposure duration and task. Brain. 117:1255-1269.

Ratcliff R, Rouder JF. 1998. Modeling response times for two-choice decisions. Psychol Sci. 9:347-356.

Rumsey JM, Horwitz B, Donohue BC, Nace K, Maisog JM, Andreason P. 1997. Phonological and orthographic components of word recognition. Brain. 120:739-759.

Salthouse TA. 1993. Speed mediation of adult age differences in cognition. Dev Psychol. 29:722-738.

Schmitt FA, Wetherby MM, Wekstein DR, Dearth CM, Markesbery WR. 2001. Brain donation in normal aging: procedures, motivations, and donor characteristics from the Biologically Resilient Adults in Neurological Studies (BRAiNS) Project. Gerontologist. 41:716-22.

Schneider W, Shiffrin RM. 1977. Controlled and automatic human information processing. I. Detection, search, and attention. Psychol Rev. 84:1-66.

Sharp DJ, Scott SK, Wise RJ. 2004. Retrieving meaning after temporal lobe infarction: the role of the basal language area. Ann Neurol. 56:836-846.

Talairach J, Tournoux P. 1988. Co-planar stereotaxic atlas of the human brain. Stuttgart (Germany): Thieme

Thompson-Schill SL, D'Esposito M, Aguirre GK, Farah MJ. 1997. Role of left inferior prefrontal cortex in retrieval of semantic knowledge: a reevaluation. Proc Natl Acad Sci U S A. 94:14792-14797.

Van Essen DC, Dickson J, Harwell J, Hanlon D, Anderson CH, Drury HA. 2001. An integrated software system for surface-based analyses of cerebral cortex. J Am Med Inform Assoc. 41:1359-1378.

Wheatley T, Weisberg J, Beauchamp MS, Martin A. 2005. Automatic priming of semantically related words reduces activity in the fusiform gyrus. J Cogn Neurosci. 17:1871-1885. 